\title{
Measurements of absolute branching fractions for $D$ mesons decays into two pseudoscalar mesons
}

M. Ablikim, ${ }^{1}$ M. N. Achasov, ${ }^{9, d}$ S. Ahmed, ${ }^{14}$ M. Albrecht, ${ }^{4}$ A. Amoroso,${ }^{53 a, 53 c}$ F. F. An, ${ }^{1}$ Q. An,${ }^{50,40}$ J. Z. Bai, ${ }^{1}$ Y. Bai, ${ }^{39}$ O. Bakina, ${ }^{24}$ R. Baldini Ferroli, ${ }^{20 a}$ Y. Ban ${ }^{32}$ D. W. Bennett, ${ }^{19}$ J. V. Bennett, ${ }^{5}$ N. Berger, ${ }^{23}$ M. Bertani ${ }^{20 a}$ D. Bettoni, ${ }^{21 a}$ J. M. Bian, ${ }^{47}$ F. Bianchi ${ }^{53 a, 53 c}$ E. Boger,${ }^{24, b}$ I. Boyko, ${ }^{24}$ R. A. Briere, ${ }^{5}$ H. Cai ${ }^{55}$ X. Cai ${ }^{1,40}$ O. Cakir, ${ }^{43 a}$ A. Calcaterra, ${ }^{20 a}$ G. F. Cao,${ }^{1,44}$ S. A. Cetin ${ }^{43 b}$ J. Chai ${ }^{53 c}$ J. F. Chang,${ }^{1,40}$ G. Chelkov, ${ }^{24, b, c}$ G. Chen, ${ }^{1}$ H. S. Chen, ${ }^{1,44}$ J. C. Chen, ${ }^{1}$ M. L. Chen, ${ }^{1,40}$ P. L. Chen, ${ }^{51}$ S. J. Chen,${ }^{30}$ X. R. Chen, ${ }^{27}$ Y. B. Chen,${ }^{1,40}$ X. K. Chu,${ }^{32}$ G. Cibinetto, ${ }^{21 a}$ H. L. Dai, ${ }^{1,40}$ J. P. Dai, ${ }^{35, h}$ A. Dbeyssi,${ }^{14}$ D. Dedovich, ${ }^{24}$ Z. Y. Deng, ${ }^{1}$ A. Denig, ${ }^{23}$ I. Denysenko, ${ }^{24}$ M. Destefanis,${ }^{53 a, 53 c}$ F. De Mori,${ }^{53 a, 53 c}$ Y. Ding, ${ }^{28}$ C. Dong, ${ }^{31}$ J. Dong, ${ }^{1,40}$ L. Y. Dong, ${ }^{1,44}$ M. Y. Dong, ${ }^{1,40,44}$ Z. L. Dou, ${ }^{30}$ S. X. Du, ${ }^{57}$ P. F. Duan, ${ }^{1}$ J. Fang, ${ }^{1,40}$ S. S. Fang, ${ }^{1,44}$ Y. Fang, ${ }^{1}$ R. Farinelli, ${ }^{21 a, 21 b}$ L. Fava, ${ }^{53 b, 53 c}$ S. Fegan, ${ }^{23}$ F. Feldbauer, ${ }^{23}$ G. Felici, ${ }^{20 a}$ C. Q. Feng, ${ }^{50,40}$ E. Fioravanti, ${ }^{21 a}$ M. Fritsch, ${ }^{23,14}$ C. D. Fu, ${ }^{1}$ Q. Gao, ${ }^{1}$ X. L. Gao ${ }^{50,40}$ Y. Gao, ${ }^{42}$ Y. G. Gao, ${ }^{6}$ Z. Gao,${ }^{50,40}$ I. Garzia,${ }^{21 a}$ K. Goetzen, ${ }^{10}$ L. Gong, ${ }^{31}$ W. X. Gong, ${ }^{1,40}$ W. Gradl, ${ }^{23}$ M. Greco,${ }^{53 a, 53 c}$ M. H. Gu, ${ }^{1,40}$ Y. T. Gu, ${ }^{12}$ A. Q. Guo, ${ }^{1}$ R. P. Guo, ${ }^{1,44}$ Y. P. Guo, ${ }^{23}$ Z. Haddadi, ${ }^{26}$ S. Han, ${ }^{55}$ X. Q. Hao ${ }^{15}$ F. A. Harris, ${ }^{45}$ K. L. He,${ }^{1,44}$ X. Q. He,${ }^{49}$ F. H. Heinsius, ${ }^{4}$ T. Held, ${ }^{4}$ Y. K. Heng, ${ }^{1,40,44}$ T. Holtmann ${ }^{4}$ Z. L. Hou, ${ }^{1}$ H. M. Hu, ${ }^{1,44}$ T. Hu, ${ }^{1,40,44}$ Y. Hu, ${ }^{1}$ G. S. Huang,${ }^{50,40}$ J. S. Huang, ${ }^{15}$ X. T. Huang, ${ }^{34}$ X. Z. Huang, ${ }^{30}$ Z. L. Huang, ${ }^{28}$ T. Hussain ${ }^{52}$ W. Ikegami Andersson, ${ }^{54}$ Q. Ji, ${ }^{1}$ Q. P. Ji, ${ }^{15}$ X. B. Ji, ${ }^{1,44}$ X. L. Ji, ${ }^{1,40}$ X. S. Jiang,,${ }^{1,40,44}$ X. Y. Jiang, ${ }^{31}$ J. B. Jiao, ${ }^{34}$ Z. Jiao ${ }^{17}$

D. P. Jin, ${ }^{1,40,44}$ S. Jin, ${ }^{1,44}$ Y. Jin, ${ }^{46}$ T. Johansson, ${ }^{54}$ A. Julin, ${ }^{47}$ N. Kalantar-Nayestanaki, ${ }^{26}$ X. L. Kang, ${ }^{1}$ X. S. Kang, ${ }^{31}$

M. Kavatsyuk, ${ }^{26}$ B. C. Ke, ${ }^{5}$ T. Khan,${ }^{50,40}$ A. Khoukaz,${ }^{48}$ P. Kiese, ${ }^{23}$ R. Kliemt,${ }^{10}$ L. Koch,${ }^{25}$ O. B. Kolcu, ${ }^{43 b, f}$ B. Kopf, ${ }^{4}$ M. Kornicer ${ }^{45}$ M. Kuemmel, ${ }^{4}$ M. Kuessner, ${ }^{4}$ M. Kuhlmann, ${ }^{4}$ A. Kupsc, ${ }^{54}$ W. Kühn, ${ }^{25}$ J. S. Lange,${ }^{25}$ M. Lara, ${ }^{19}$ P. Larin,${ }^{14}$ L. Lavezzi, ${ }^{53}$ H. Leithoff, ${ }^{23}$ C. Leng, ${ }^{53 c}$ C. Li ${ }^{54}$ Cheng Li ${ }^{50,40}$ D. M. Li, ${ }^{57}$ F. Li, ${ }^{1,40}$ F. Y. Li ${ }^{32}$ G. Li ${ }^{1}{ }^{1}$ H. B. Li,${ }^{1,44}$ H. J. Li, ${ }^{1,44}$

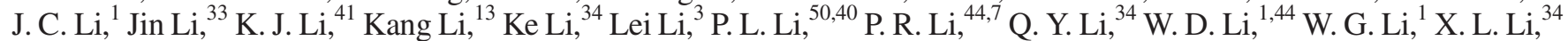
X. N. Li ${ }^{1,40}$ X. Q. Li, ${ }^{31}$ Z. B. Li ${ }^{41}$ H. Liang, ${ }^{50,40}$ Y. F. Liang, ${ }^{37}$ Y. T. Liang, ${ }^{25}$ G. R. Liao, ${ }^{11}$ D. X. Lin, ${ }^{14}$ B. Liu,${ }^{35}$, B. J. Liu, ${ }^{1}$ C. X. Liu, ${ }^{1}$ D. Liu, ${ }^{50,40}$ F. H. Liu, ${ }^{36}$ Fang Liu, ${ }^{1}$ Feng Liu ${ }^{6}$ H. B. Liu, ${ }^{12}$ H. M. Liu, ${ }^{1,44}$ Huanhuan Liu, ${ }_{1}^{1}$ Huihui Liu,${ }^{16}$ J. B. Liu, ${ }^{50,40}$ J. P. Liu,${ }^{55}$ J. Y. Liu, ${ }^{1,44}$ K. Liu, ${ }^{42}$ K. Y. Liu, ${ }^{28}$ Ke Liu, ${ }^{6}$ L. D. Liu ${ }^{32}$ P. L. Liu,${ }^{1,40}$ Q. Liu, ${ }^{44}$ S. B. Liu, ${ }^{50,40}$ X. Liu, ${ }^{27}$ Y. B. Liu, ${ }^{31}$

Z. A. Liu, ${ }^{1,40,44}$ Zhiqing Liu, ${ }^{23}$ Y. F. Long, ${ }^{32}$ X. C. Lou, ${ }^{1,40,44}$ H. J. Lu, ${ }^{17}$ J. G. Lu, ${ }^{1,40}$ Y. Lu, ${ }^{1}$ Y. P. Lu, ${ }^{1,40}$ C. L. Luo, ${ }^{29}$

M. X. Luo, ${ }^{56}$ X. L. Luo, ${ }^{1,40}$ X. R. Lyu ${ }^{44}$ F. C. Ma, ${ }^{28}$ H. L. Ma, ${ }^{1}$ L. L. Ma, ${ }^{34}$ M. M. Ma, ${ }^{1,44}$ Q. M. Ma, ${ }^{1}$ T. Ma, ${ }^{1}$ X. N. Ma, ${ }^{31}$

X. Y. Ma, ${ }^{1,40}$ Y. M. Ma,${ }^{34}$ F. E. Maas, ${ }^{14}$ M. Maggiora, ${ }^{53,53 c}$ Q. A. Malik ${ }^{52}$ Y. J. Mao, ${ }^{32}$ Z. P. Mao, ${ }^{1}$ S. Marcello, ${ }^{53 a, 53 c}$

Z. X. Meng, ${ }^{46}$ J. G. Messchendorp, ${ }^{26}$ G. Mezzadri, ${ }^{21 b}$ J. Min, ${ }^{1,40}$ T. J. Min, ${ }^{1}$ R. E. Mitchell, ${ }^{19}$ X. H. Mo, ${ }^{1,40,44}$ Y. J. Mo, ${ }^{6}$

C. Morales Morales, ${ }^{14}$ N. Yu. Muchnoi, ${ }^{9, d}$ H. Muramatsu, ${ }^{47}$ A. Mustafa ${ }^{4}$ Y. Nefedov,${ }^{24}$ F. Nerling, ${ }^{10}$ I. B. Nikolaev, ${ }^{9, d}$

Z. Ning, ${ }^{1,40}$ S. Nisar, ${ }^{8}$ S. L. Niu, ${ }^{1,40}$ X. Y. Niu, ${ }^{1,44}$ S. L. Olsen, ${ }^{33, j}$ Q. Ouyang, ${ }^{1,40,44}$ S. Pacetti, ${ }^{20 b}$ Y. Pan,${ }^{50,40}$ M. Papenbrock, ${ }^{54}$

P. Patteri ${ }^{20 a}$ M. Pelizaeus, ${ }^{4}$ J. Pellegrino, ${ }^{53 a, 53 c}$ H. P. Peng,${ }^{50,40}$ K. Peters, ${ }^{10, g}$ J. Pettersson, ${ }^{54}$ J. L. Ping,${ }^{29}$ R. G. Ping, ${ }^{1,44}$

A. Pitka, ${ }^{23}$ R. Poling, ${ }^{47}$ V. Prasad, ${ }^{50,40}$ H. R. Qi, ${ }^{2}$ M. Qi ${ }^{30}$ S. Qian, ${ }^{1,40}$ C. F. Qiao, ${ }^{44}$ N. Qin, ${ }^{55}$ X. S. Qin, Z. H. Qin, ${ }^{1,40}$ J. F. Qiu, ${ }^{1}$ K. H. Rashid,${ }^{52, \mathrm{i}}$ C. F. Redmer ${ }^{23}$ M. Richter, ${ }^{4}$ M. Ripka ${ }^{23}$ M. Rolo,${ }^{53 \mathrm{c}}$ G. Rong,${ }^{1,44}$ Ch. Rosner, ${ }^{14}$ A. Sarantsev,${ }^{24, \mathrm{e}}$ M. Savrié, ${ }^{21 \mathrm{~b}}$ C. Schnier, ${ }^{4}$ K. Schoenning, ${ }^{54}$ W. Shan,${ }^{32}$ M. Shao,${ }^{50,40}$ C. P. Shen, ${ }^{2}$ P. X. Shen, ${ }^{31}$ X. Y. Shen, ${ }^{1,44}$ H. Y. Sheng, ${ }^{1}$ J. J. Song, ${ }^{34}$

W. M. Song ${ }^{34}$ X. Y. Song, ${ }^{1}$ S. Sosio,${ }^{53 a, 53 c}$ C. Sowa, ${ }^{4}$ S. Spataro, ${ }^{53 a, 53 c}$ G. X. Sun, ${ }^{1}$ J. F. Sun, ${ }^{15}$ L. Sun, ${ }^{55}$ S. S. Sun, ${ }^{1,44}$ X. H. Sun, ${ }^{1}$ Y. J. Sun,${ }^{50,40}$ Y. K. Sun, ${ }^{50,40}$ Y. Z. Sun, ${ }^{1}$ Z. J. Sun, ${ }^{1,40}$ Z. T. Sun, ${ }^{19}$ C. J. Tang, ${ }^{37}$ G. Y. Tang,,${ }^{1}$ X. Tang, ${ }^{1}$ I. Tapan, ${ }^{43 c}$ M. Tiemens, ${ }^{26}$ B. Tsednee, ${ }^{22}$ I. Uman, ${ }^{43 \mathrm{~d}}$ G. S. Varner, ${ }^{45}$ B. Wang, ${ }^{1}$ B. L. Wang ${ }^{44}$ D. Wang, ${ }^{32}$ D. Y. Wang ${ }^{32}$ Dan Wang, ${ }^{44}$ K. Wang ${ }^{1,40}$ L. L. Wang, ${ }^{1}$ L. S. Wang, ${ }^{1}$ M. Wang, ${ }^{34}$ Meng Wang, ${ }^{1,44}$ P. Wang, ${ }^{1}$ P. L. Wang, ${ }^{1}$ W. P. Wang, ${ }^{50,40}$ X. F. Wang ${ }^{42}$ Y. Wang, ${ }^{38}$ Y. D. Wang, ${ }^{14}$ Y. F. Wang, ${ }^{1,40,44}$ Y. Q. Wang, ${ }^{23}$ Z. Wang, ${ }^{1,40}$ Z. G. Wang, ${ }^{1,40}$ Z. Y. Wang, ${ }^{1}$ Zongyuan Wang, ${ }^{1,44}$ T. Weber, ${ }^{23}$ D. H. Wei, ${ }^{11}$ J. H. Wei, ${ }^{31,}{ }^{,}$P. Weidenkaff, ${ }^{23}$ S. P. Wen, ${ }^{1}$ U. Wiedner, ${ }^{4}$ M. Wolke, ${ }^{54}$ L. H. Wu, ${ }^{1}$ L. J. Wu, ${ }^{1,44}$ Z. Wu, ${ }^{1,40}$ L. Xia ${ }^{50,40}$ Y. Xia,${ }^{18}$ D. Xiao, ${ }^{1}$ H. Xiao, ${ }^{51}$ Y. J. Xiao, ${ }^{1,44}$ Z. J. Xiao, ${ }^{29}$ Y. G. Xie, ${ }^{1,40}$ Y. H. Xie, ${ }^{6}$ X. A. Xiong, ${ }^{1,44}$ Q. L. Xiu, ${ }^{1,40}$ G. F. Xu, ${ }^{1}$ J. J. Xu, ${ }^{1,44}$ L. Xu, ${ }^{1}$ Q. J. Xu, ${ }^{13}$ Q. N. Xu, ${ }^{44}$ X. P. Xu, ${ }^{38}$ L. Yan, ${ }^{53,53 c}$ W. B. Yan, ${ }^{50,40}$ W. C. Yan, ${ }^{2}$ Y. H. Yan, ${ }^{18}$ H. J. Yang, ${ }^{35, h}$ H. X. Yang, ${ }^{1}$ L. Yang,${ }^{55}$ Y. H. Yang, ${ }^{30}$ Y. X. Yang, ${ }^{11}$ M. Ye ${ }^{1,40}$ M. H. Ye,${ }^{7}$ J. H. Yin, ${ }^{1}$ Z. Y. You, ${ }^{41}$ B. X. Yu,${ }^{1,40,44}$ C. X. Yu, ${ }^{31}$ J. S. Yu, ${ }^{27}$ C. Z. Yuan, ${ }^{1,44}$ Y. Yuan, ${ }^{1}$ A. Yuncu, ${ }^{43 b, a}$ A. A. Zafar, ${ }^{52}$ Y. Zeng, ${ }^{18}$ Z. Zeng, ${ }^{50,40}$ B. X. Zhang, ${ }^{1}$ B. Y. Zhang, ${ }^{1,40}$ C. C. Zhang, ${ }^{1}$ D. H. Zhang, ${ }^{1}$ H. H. Zhang, ${ }^{41}$ H. Y. Zhang, ${ }^{1,40}$ J. Zhang, ${ }^{1,44}$ J. L. Zhang, ${ }^{1}$ J. Q. Zhang, ${ }^{1}$

J. W. Zhang, ${ }^{1,40,44}$ J. Y. Zhang, ${ }^{1}$ J. Z. Zhang, ${ }^{1,44}$ K. Zhang, ${ }^{1,44}$ L. Zhang, ${ }^{42}$ S. Q. Zhang, ${ }^{31}$ X. Y. Zhang, ${ }^{34}$ Y. H. Zhang, ${ }^{1,40}$ Y. T. Zhang, ${ }^{50,40}$ Yang Zhang, ${ }^{1}$ Yao Zhang, ${ }^{1}$ Yu Zhang, ${ }^{44}$ Z. H. Zhang, ${ }^{6}$ Z. P. Zhang, ${ }^{50}$ Z. Y. Zhang, ${ }^{55}$ G. Zhao, ${ }^{1}$ J. W. Zhao, ${ }^{1,40}$ J. Y. Zhao, ${ }^{1,44}$ J. Z. Zhao, ${ }^{1,40}$ Lei Zhao, ${ }^{50,40}$ Ling Zhao, ${ }^{1}$ M. G. Zhao, ${ }^{31, \dagger}$ Q. Zhao, ${ }^{1}$ S. J. Zhao, ${ }^{57}$ T. C. Zhao, ${ }^{1}$ Y. B. Zhao, ${ }^{1,40}$

Z. G. Zhao, ${ }^{50,40}$ A. Zhemchugov, ${ }^{24, b}$ B. Zheng, ${ }^{51}$ J. P. Zheng, ${ }^{1,40}$ Y. H. Zheng, ${ }^{44}$ B. Zhong, ${ }^{29}$ L. Zhou, ${ }^{1,40}$ X. Zhou, ${ }^{55}$ X. K. Zhou, ${ }^{50,40}$ X. R. Zhou, ${ }^{50,40}$ X. Y. Zhou, ${ }^{1}$ J. Zhu, ${ }^{31}$ J. Zhu ${ }^{41}$ K. Zhu, ${ }^{1}$ K. J. Zhu, ${ }^{1,40,44}$ S. Zhu, ${ }^{1}$ S. H. Zhu, ${ }^{49}$ X. L. Zhu, ${ }^{42}$ Y. C. Zhu, ${ }^{50,40}$ Y. S. Zhu, ${ }^{1,44}$ Z. A. Zhu, ${ }^{1,44}$ J. Zhuang, ${ }^{1,40}$ B. S. Zou, ${ }^{1}$ and J. H. Zou ${ }^{1}$

(BESIII Collaboration) 
${ }^{1}$ Institute of High Energy Physics, Beijing 100049, People's Republic of China

${ }^{2}$ Beihang University, Beijing 100191, People's Republic of China

${ }^{3}$ Beijing Institute of Petrochemical Technology, Beijing 102617, People's Republic of China

${ }^{4}$ Bochum Ruhr-University, D-44780 Bochum, Germany

${ }^{5}$ Carnegie Mellon University, Pittsburgh, Pennsylvania 15213, USA

${ }^{6}$ Central China Normal University, Wuhan 430079, People's Republic of China

${ }^{7}$ China Center of Advanced Science and Technology, Beijing 100190, People's Republic of China

${ }^{8}$ COMSATS Institute of Information Technology, Lahore, Defence Road, Off Raiwind Road, 54000 Lahore, Pakistan

${ }^{9}$ G.I. Budker Institute of Nuclear Physics SB RAS (BINP), Novosibirsk 630090, Russia

${ }^{10}$ GSI Helmholtzcentre for Heavy Ion Research GmbH, D-64291 Darmstadt, Germany

${ }^{11}$ Guangxi Normal University, Guilin 541004, People's Republic of China

${ }^{12}$ Guangxi University, Nanning 530004, People's Republic of China

${ }^{13}$ Hangzhou Normal University, Hangzhou 310036, People's Republic of China

${ }^{14}$ Helmholtz, Institute Mainz, Johann-Joachim-Becher-Weg 45, D-55099 Mainz, Germany

${ }^{15}$ Henan Normal University, Xinxiang 453007, People's Republic of China

${ }^{16}$ Henan University of Science and Technology, Luoyang 471003, People's Republic of China

${ }^{17}$ Huangshan College, Huangshan 245000, People's Republic of China

${ }^{18}$ Hunan University, Changsha 410082, People's Republic of China

${ }^{19}$ Indiana University, Bloomington, Indiana 47405, USA

${ }^{20 a}$ INFN Laboratori Nazionali di Frascati, I-00044 Frascati, Italy

${ }^{20 \mathrm{~b}}$ INFN and University of Perugia, I-06100 Perugia, Italy

${ }^{21 a}$ INFN Sezione di Ferrara, I-44122 Ferrara, Italy

${ }^{21 \mathrm{~b}}$ University of Ferrara, I-44122 Ferrara, Italy

${ }^{22}$ Institute of Physics and Technology, Peace Ave. 54B, Ulaanbaatar 13330, Mongolia

${ }^{23}$ Johannes Gutenberg University of Mainz, Johann-Joachim-Becher-Weg 45, D-55099 Mainz, Germany

${ }^{24}$ Joint Institute for Nuclear Research, 141980 Dubna, Moscow region, Russia

${ }^{25}$ Justus-Liebig-Universitaet Giessen, II. Physikalisches Institut, Heinrich-Buff-Ring 16, D-35392 Giessen, Germany

${ }^{26}$ KVI-CART, University of Groningen, NL-9747 AA Groningen, The Netherlands

${ }^{27}$ Lanzhou University, Lanzhou 730000, People's Republic of China

${ }^{28}$ Liaoning University, Shenyang 110036, People's Republic of China

${ }^{29}$ Nanjing Normal University, Nanjing 210023, People's Republic of China

${ }^{30}$ Nanjing University, Nanjing 210093, People's Republic of China

${ }^{31}$ Nankai University, Tianjin 300071, People's Republic of China

${ }^{32}$ Peking University, Beijing 100871, People's Republic of China

${ }^{33}$ Seoul National University, Seoul, 151-747 Korea

${ }^{34}$ Shandong University, Jinan 250100, People's Republic of China

${ }^{35}$ Shanghai Jiao Tong University, Shanghai 200240, People's Republic of China

${ }^{36}$ Shanxi University, Taiyuan 030006, People's Republic of China

${ }^{37}$ Sichuan University, Chengdu 610064, People's Republic of China

${ }^{38}$ Soochow University, Suzhou 215006, People's Republic of China

${ }^{39}$ Southeast University, Nanjing 211100, People's Republic of China

${ }^{40}$ State Key Laboratory of Particle Detection and Electronics, Beijing 100049, Hefei 230026, People's Republic of China

${ }^{41}$ Sun Yat-Sen University, Guangzhou 510275, People's Republic of China

${ }^{42}$ Tsinghua University, Beijing 100084, People's Republic of China

${ }^{43 a}$ Ankara University, 06100 Tandogan, Ankara, Turkey

${ }^{43 \mathrm{~b}}$ Istanbul Bilgi University, 34060 Eyup, Istanbul, Turkey

${ }^{43 \mathrm{c}}$ Uludag University, 16059 Bursa, Turkey

${ }^{43 \mathrm{~d}}$ Near East University, Nicosia, North Cyprus, Mersin 10, Turkey

${ }^{44}$ University of Chinese Academy of Sciences, Beijing 100049, People's Republic of China

${ }^{45}$ University of Hawaii, Honolulu, Hawaii 96822, USA

${ }^{46}$ University of Jinan, Jinan 250022, People's Republic of China

${ }^{47}$ University of Minnesota, Minneapolis, Minnesota 55455, USA

${ }^{48}$ University of Muenster, Wilhelm-Klemm-Str. 9, 48149 Muenster, Germany

${ }^{49}$ University of Science and Technology Liaoning, Anshan 114051, People's Republic of China

${ }^{50}$ University of Science and Technology of China, Hefei 230026, People's Republic of China

${ }^{51}$ University of South China, Hengyang 421001, People's Republic of China

${ }^{52}$ University of the Punjab, Lahore-54590, Pakistan 


\author{
${ }^{53 a}$ University of Turin, I-10125, Turin, Italy \\ ${ }^{53 \mathrm{~b}}$ University of Eastern Piedmont, I-15121, Alessandria, Italy \\ ${ }^{53 \mathrm{c}}$ INFN, I-10125, Turin, Italy \\ ${ }^{54}$ Uppsala University, Box 516, SE-75120 Uppsala, Sweden \\ ${ }^{55}$ Wuhan University, Wuhan 430072, People's Republic of China \\ ${ }^{56}$ Zhejiang University, Hangzhou 310027, People's Republic of China \\ ${ }^{57}$ Zhengzhou University, Zhengzhou 450001, People's Republic of China
}

(Received 8 February 2018; published 9 April 2018)

\begin{abstract}
Using a data sample of $e^{+} e^{-}$collision data with an integrated luminosity of $2.93 \mathrm{fb}^{-1}$ taken at the centerof-mass energy $\sqrt{s}=3.773 \mathrm{GeV}$ with the BESIII detector operating at the BEPCII storage rings, we measure the absolute branching fractions of the two-body hadronic decays $D^{+} \rightarrow \pi^{+} \pi^{0}, K^{+} \pi^{0}, \pi^{+} \eta, K^{+} \eta$, $\pi^{+} \eta^{\prime}, K^{+} \eta^{\prime}, K_{S}^{0} \pi^{+}, K_{S}^{0} K^{+}$, and $D^{0} \rightarrow \pi^{+} \pi^{-}, K^{+} K^{-}, K^{\mp} \pi^{ \pm}, K_{S}^{0} \pi^{0}, K_{S}^{0} \eta, K_{S}^{0} \eta^{\prime}$. Our results are consistent with previous measurements within uncertainties. Among them, the branching fractions for $D^{+} \rightarrow \pi^{+} \pi^{0}$, $K^{+} \pi^{0}, \pi^{+} \eta, \pi^{+} \eta^{\prime}, K_{S}^{0} \pi^{+}, K_{S}^{0} K^{+}$and $D^{0} \rightarrow K_{S}^{0} \pi^{0}, K_{S}^{0} \eta, K_{S}^{0} \eta^{\prime}$ are determined with improved precision compared to the world average values.
\end{abstract}

DOI: 10.1103/PhysRevD.97.072004

\section{INTRODUCTION}

The two-body hadronic decays $D \rightarrow P_{1} P_{2}$ (throughout the text, $D$ represents the $D^{+}$and $D^{0}$ mesons and $P$ denotes one of the pseudoscalar mesons $\pi^{ \pm}, K^{ \pm}, K_{S}^{0}, \pi^{0}, \eta$ and $\eta^{\prime}$ ) serve as an ideal test bed to improve the understanding of the weak and strong interactions in decays of charmed mesons. These reactions proceed via external $W$-emission, internal $W$-emission or $W$-exchange processes. Due to the relatively simple topology, the amplitude of $D \rightarrow P_{1} P_{2}$ decay can be theoretically derived as a sum of different diagrams based on

\footnotetext{
*weijh@mail.nankai.edu.cn

'zhaomg@nankai.edu.cn

${ }^{\mathrm{a}}$ Also at Bogazici University, 34342 Istanbul, Turkey.

${ }^{b}$ Also at the Moscow Institute of Physics and Technology, Moscow 141700, Russia.

${ }^{\mathrm{c}}$ Also at the Functional Electronics Laboratory, Tomsk State University, Tomsk, 634050, Russia.

${ }^{\mathrm{d}}$ Also at the Novosibirsk State University, Novosibirsk, 630090, Russia.

"Also at the NRC "Kurchatov Institute", PNPI, 188300, Gatchina, Russia.

${ }^{\mathrm{f}}$ Also at Istanbul Arel University, 34295 Istanbul, Turkey.

${ }^{\mathrm{g}}$ Also at Goethe University Frankfurt, 60323 Frankfurt am Main, Germany.

${ }^{\mathrm{h}}$ Also at Key Laboratory for Particle Physics, Astrophysics and Cosmology, Ministry of Education; Shanghai Key Laboratory for Particle Physics and Cosmology; Institute of Nuclear and Particle Physics, Shanghai 200240, People's Republic of China.

${ }^{1}$ Government College Women University, Sialkot-51310. Punjab, Pakistan.

${ }^{\mathrm{j}}$ Present address: Center for Underground Physics, Institute for Basic Science, Daejeon 34126, Korea.

Published by the American Physical Society under the terms of the Creative Commons Attribution 4.0 International license. Further distribution of this work must maintain attribution to the author(s) and the published article's title, journal citation, and DOI. Funded by SCOAP ${ }^{3}$.
}

SU(3)-flavor symmetry [1]. Comprehensive and improved experimental measurements of the branching fractions for these decays may help to validate the theoretical calculations and provide important and complementary data to explore the effect of SU(3)-flavor symmetry breaking in hadronic decays of the $D$ mesons [2-5].

Historically, experimental studies of singly or doublyCabibbo-suppressed (DCS) decays of $D \rightarrow P_{1} P_{2}$ with branching fractions at the $10^{-4}$ level were challenging due to limited statistics and high background. In recent years, the $D \rightarrow P_{1} P_{2}$ decays have been widely studied in various experiments [6-10]. The BESIII Collaboration has recently reported measurements of the branching fractions for some $D \rightarrow P_{1} P_{2}$ decays [11-14] by analyzing the data sample corresponding to an integrated luminosity of $2.93 \mathrm{fb}^{-1}$ [15] taken at the center-of-mass energy $\sqrt{s}=3.773 \mathrm{GeV}$. Singletag or double-tag methods, in which one or two $D$ mesons are fully reconstructed, have been used in previous works. Analyzing the same data sample with the single-tag method, we report in this paper the measurements of the absolute branching fractions of the two-body hadronic decays $D^{+} \rightarrow \pi^{+} \pi^{0}, K^{+} \pi^{0}, \pi^{+} \eta, K^{+} \eta, \pi^{+} \eta^{\prime}, K^{+} \eta^{\prime}, K_{S}^{0} \pi^{+}, K_{S}^{0} K^{+}$, and $D^{0} \rightarrow \pi^{+} \pi^{-}, K^{+} K^{-}, K^{\mp} \pi^{ \pm}, K_{S}^{0} \pi^{0}, K_{S}^{0} \eta, K_{S}^{0} \eta^{\prime}$, where $D^{0} \rightarrow K^{\mp} \pi^{ \pm}$includes both the Cabibbo-favored decay of $D^{0} \rightarrow K^{-} \pi^{+}$and the DCS decay of $D^{0} \rightarrow K^{+} \pi^{-}$. Throughout this paper, charge-conjugated modes are implied.

\section{BESIII DETECTOR AND MONTE CARLO SIMULATION}

The BESIII detector is a cylindrical detector with a solidangle coverage of $93 \%$ of $4 \pi$ that operates at the BEPCII collider. It consists of several main components. A 43-layer main drift chamber (MDC) surrounding the beam pipe 
performs precise determinations of charged particle trajectories and provides a measurement of ionization energy loss $(d E / d x)$ that is used for charged particle identification (PID). An array of time-of-flight counters (TOF) is located outside the MDC and provides further information for PID. A CsI(Tl) electromagnetic calorimeter (EMC) surrounds the TOF and is used to measure the energies of photons and electrons. A solenoidal superconducting magnet outside the EMC provides a $1 \mathrm{~T}$ magnetic field in the central tracking region of the detector. The iron flux return yoke of the magnet is instrumented with about $1272 \mathrm{~m}^{2}$ resistive plate muon counters, arranged in nine layers in the barrel and eight layers in the end caps, that are used to identify muons with momenta greater than $0.5 \mathrm{GeV} / c$. More details about the BESIII detector are described in Ref. [16].

A GEANT4-based [17] Monte Carlo (MC) simulation software package, which includes the geometric description of the detector and its response, is used to determine the detection efficiency and to estimate the potential background. An inclusive MC sample, which includes $D^{0} \bar{D}^{0}$, $D^{+} D^{-}$and non- $D \bar{D}$ decays of the $\psi(3770)$, initial-state radiation (ISR) production of the $\psi(3686)$ and $J / \psi$, $e^{+} e^{-} \rightarrow q \bar{q}(q=u, d, s)$ continuum processes, Bhabha scattering events, dimuon events and ditau events, is produced at $\sqrt{s}=3.773 \mathrm{GeV}$. The $\psi(3770)$ production is simulated by the MC generator KKMC [18], in which the effects of ISR [19] and final-state radiation [20] are considered. The known decay modes are generated using EvTGEN [21] with the branching fractions taken from the Particle Data Group (PDG) [22], and unknown decay modes are generated using LUNDCHARM [23].

\section{DATA ANALYSIS}

The $D$ meson candidates are selected from combinations of $\pi^{ \pm}, K^{ \pm}, K_{S}^{0}, \pi^{0}, \eta$ and $\eta^{\prime}$, where $K_{S}^{0}, \pi^{0}, \eta$ and $\eta^{\prime}$ are reconstructed through their prominent decays $K_{S}^{0} \rightarrow \pi^{+} \pi^{-}$, $\pi^{0} \rightarrow \gamma \gamma, \eta \rightarrow \gamma \gamma$ and $\eta^{\prime} \rightarrow \pi^{+} \pi^{-} \eta$, respectively.

All charged tracks, except for those from a $K_{S}^{0}$ decay, are required to originate from the interaction region defined as $V_{x y}<1 \mathrm{~cm}$ and $\left|V_{z}\right|<10 \mathrm{~cm}$, where $V_{x y}$ and $\left|V_{z}\right|$ denote the distances of the closest approach of the reconstructed track to the interaction point in the $x y$ plane and in the $z$ direction (along the beam direction), respectively. The polar angle of the charged tracks $\theta$ is required to satisfy $|\cos \theta|<0.93$. Charged tracks are identified using confidence levels for the kaon (pion) hypothesis $C L_{K(\pi)}$, calculated with both $d E / d x$ and TOF information. The kaon (pion) candidates are required to satisfy $C L_{K(\pi)}>$ $C L_{\pi(K)}$ and $C L_{K(\pi)}>0$. In the momentum range of $(0.1,0.6) \mathrm{GeV} / c$, the PID efficiencies of $\pi^{ \pm}$and $K^{ \pm}$are all greater than $99 \%$, while the misidentification rates between $\pi^{ \pm}$and $K^{ \pm}$are less than $0.8 \%$. In the momentum range of $(0.6,1.1) \mathrm{GeV} / c$, however, the PID efficiencies of $\pi^{ \pm}$and $K^{ \pm}$range in (98-94)\% and (98-90)\%, respectively, while the rates of misidentifying $\pi^{ \pm}$as $K^{ \pm}$and $K^{ \pm}$as $\pi^{ \pm}$ range in $(1-10) \%$ and $(1-6) \%$, respectively.

The $K_{S}^{0}$ candidates are formed from two oppositely charged tracks with $\left|V_{z}\right|<20 \mathrm{~cm}$ and $|\cos \theta|<0.93$. The two charged tracks are assumed to be a $\pi^{+} \pi^{-}$pair without PID and are constrained to originate from a common decay vertex. To suppress the $\pi^{+} \pi^{-}$combinatorial background, the reconstructed decay length of the $K_{S}^{0}$ candidate is required to be greater than twice its uncertainty. The $\pi^{+} \pi^{-}$invariant mass must be within the signal region, defined as $\pm 0.012 \mathrm{GeV} / c^{2}$ around the $K_{S}^{0}$ nominal mass [10].

The photon candidates are selected from isolated EMC clusters. To suppress the electronics noise and beam background, the clusters are required to start within 700 ns after the event start time and fall outside a cone angle of $10^{\circ}$ around the nearest extrapolated charged track. The minimum energy of each EMC cluster is required to be larger than $25 \mathrm{MeV}$ in the barrel region $(|\cos \theta|<0.80)$ or $50 \mathrm{MeV}$ in the end-cap region $(0.86<|\cos \theta|<0.92)$ [16]. To select the $\pi^{0}$ and $\eta$ meson candidates, the $\gamma \gamma$ invariant mass is required to be within $(0.115,0.150) \mathrm{GeV} / c^{2}$ and $(0.515,0.575) \mathrm{GeV} / c^{2}$, respectively. The momentum resolution of $\pi^{0}$ and $\eta$ is further improved with a kinematic fit that constrains the $\gamma \gamma$ invariant mass to the $\pi^{0}$ or $\eta$ nominal mass [10]. For $\eta^{\prime}$ mesons, the $\pi^{+} \pi^{-} \eta$ invariant mass is required to be within the signal region, which is $\pm 0.012 \mathrm{GeV} / c^{2}$ around the nominal $\eta^{\prime}$ mass [10].

For $D^{0}$ decays to $\pi^{+} \pi^{-}, K^{+} K^{-}$and $K^{\mp} \pi^{ \pm}$, the backgrounds arising from cosmic rays, Bhabha scattering events and dimuon events are rejected with the same requirements as those used in Ref. [24]. First, the two charged tracks must have a TOF time difference less than $5 \mathrm{~ns}$ and must not be consistent with the requirement for a muon pair or an electron-positron pair. To further suppress the backgrounds of Bhabha scattering and dimuon events, at least one EMC cluster with an energy larger than $50 \mathrm{MeV}$ or at least one additional charged track detected in the MDC is required. This requirement avoids the small kink near the beamenergy point in the $M_{\mathrm{BC}}$ (see the next paragraph for its definition) distributions.

At the $\psi(3770)$ peak, the $D \bar{D}$ meson pairs are produced without additional particles; thus, the energies of the $D$ mesons are equal to the beam energy $E_{\text {beam }}$ in the center-ofmass frame of the $e^{+} e^{-}$system. Two variables reflecting energy and momentum conservation are used to identify the $D$ meson candidates. They are the energy difference

$$
\Delta E \equiv \sum_{i} E_{i}-E_{\text {beam }}
$$

and the beam-energy-constrained mass

$$
M_{\mathrm{BC}} \cdot c^{2} \equiv \sqrt{E_{\text {beam }}^{2}-\left(\sum_{i} \vec{p}_{i} \cdot c\right)^{2}},
$$



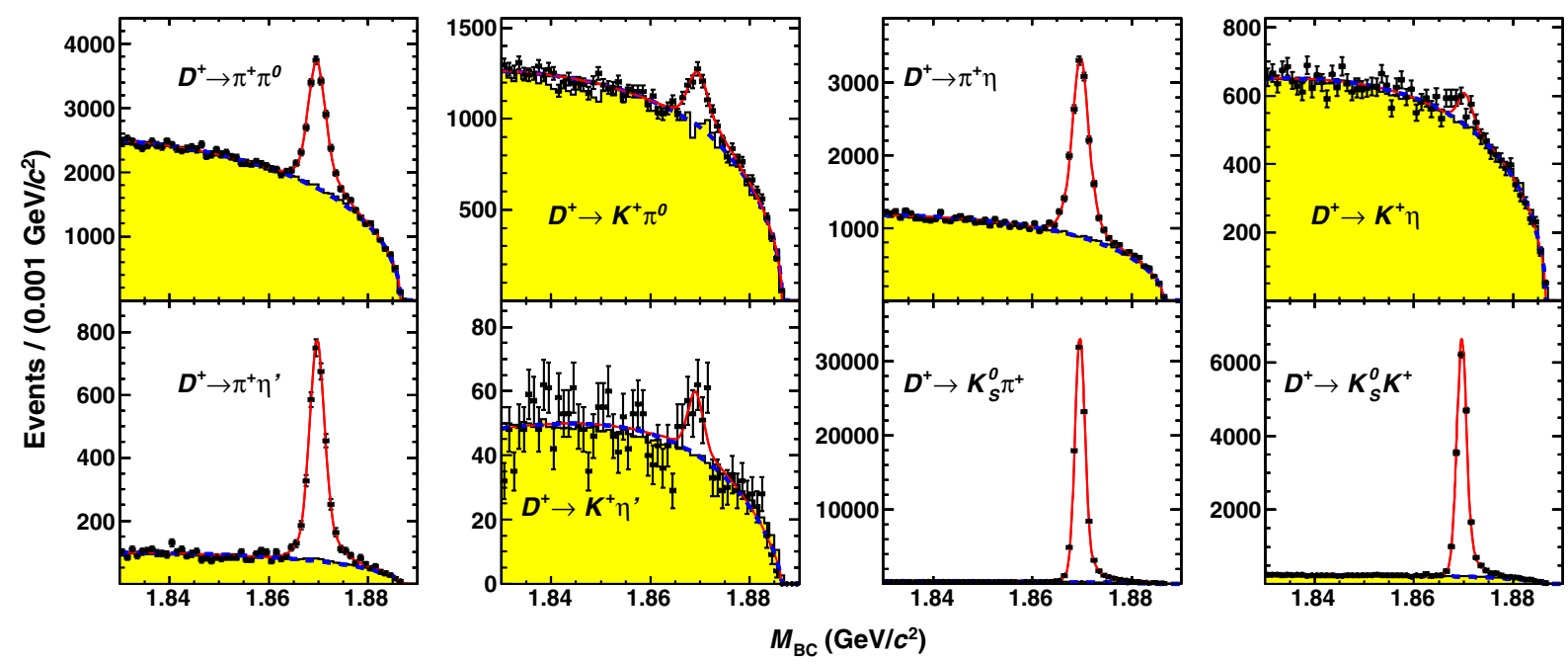

FIG. 1. Fits to the $M_{\mathrm{BC}}$ distributions of the single-tag $D^{+}$candidate events. The points with error bars are data, the red curves are the overall fits, the blue dashed curves are the fitted backgrounds and the yellow shaded histograms are the MC-simulated combinatorial backgrounds.

where $E_{i}$ and $\vec{p}_{i}$ are the energy and momentum of the decay products of the $D$ candidates in the center-of-mass frame of the $e^{+} e^{-}$system. For a given $D$ decay mode, if there is more than one candidate per tag mode per $D$ and $\bar{D}$, the one with the least $|\Delta E|$ is kept for further analysis. The combinatorial backgrounds are suppressed by modedependent $\Delta E$ requirements, which correspond to $\pm 3.0 \sigma_{\Delta E}$ around the fitted $\Delta E$ peak, where $\sigma_{\Delta E}$ is the resolution of the $\Delta E$ distribution.

Figures 1 and 2 show the $M_{\mathrm{BC}}$ distributions of the accepted single-tag $D^{+}$and $D^{0}$ candidates, respectively. The signal yields of $D$ mesons for the different processes are determined using unbinned maximum likelihood fits to the corresponding distributions, where the signal probability density function is modeled by the MC-simulated shape convolved with a double Gaussian function that describes the resolution difference between data and $\mathrm{MC}$ simulation. The combinatorial background is described with an ARGUS function [25] with the end point fixed at $E_{\text {beam }}$.

For the decays including $K_{S}^{0}\left(\eta^{\prime}\right)$ mesons in the final states, there are peaking backgrounds from non- $K_{S}^{0}$ (non- $\eta^{\prime}$ ) events in the $K_{S}^{0}\left(\eta^{\prime}\right)$ signal regions around the nominal $D$ mass in the $M_{\mathrm{BC}}$ distributions. To estimate these peaking backgrounds, the data events in the $K_{S}^{0}\left(\eta^{\prime}\right)$ sideband regions, defined as $0.020<\left|M_{\pi^{+} \pi^{-}\left(\pi^{+} \pi^{-} \eta\right)}-M_{K_{S}^{0}\left(\eta^{\prime}\right)}\right|<0.044 \mathrm{GeV} / c^{2}$, are used. Figure 3 shows the distributions of $M_{\pi^{+} \pi^{-}}, M_{\pi^{+} \pi^{-} \eta}$ as well as $M_{\pi^{+} \pi^{-}}$versus $M_{\pi^{+} \pi^{-} \eta}$ for the $D^{0} \rightarrow K_{S}^{0} \eta^{\prime}$ candidate events in data. In Figs. 3(a) and 3(b), the regions between the pair of solid (dashed) arrows denote the $K_{S}^{0}$ and $\eta^{\prime}$ signal
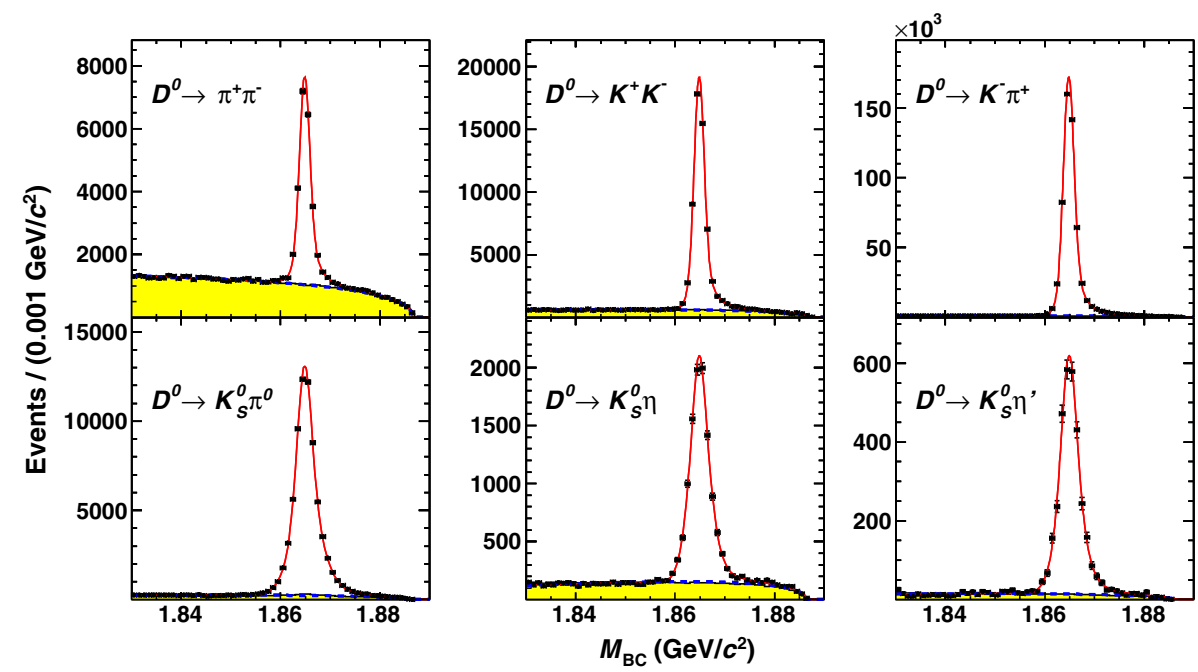

FIG. 2. Fits to the $M_{\mathrm{BC}}$ distributions of the single-tag $D^{0}$ candidate events. The points with error bars are data, the red solid curves are the overall fits, the blue dashed curves are the fitted backgrounds and the yellow shaded histograms are the MC-simulated combinatorial backgrounds. 


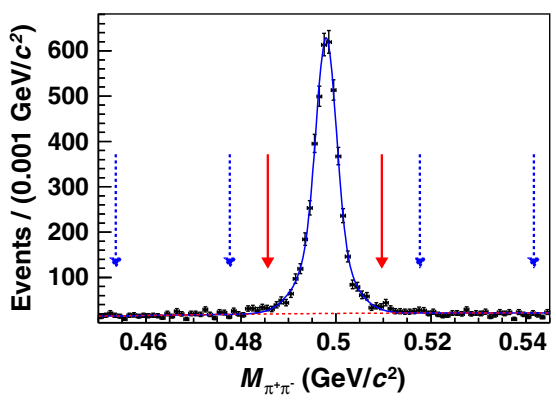

(a)

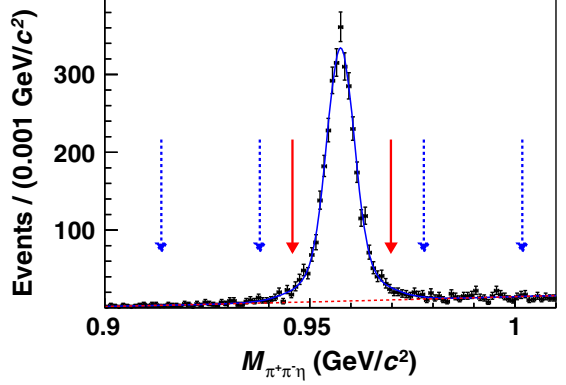

(b)

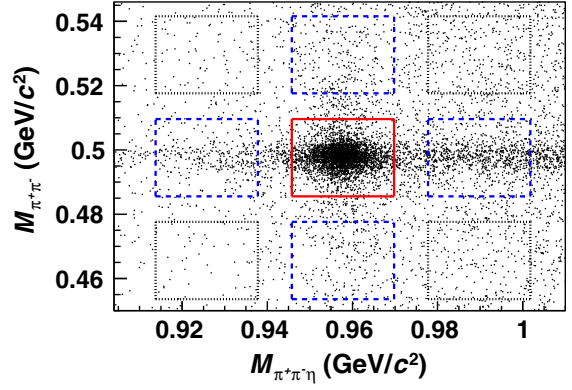

(c)

FIG. 3. Distributions of (a) $M_{\pi^{+} \pi^{-}}$, (b) $M_{\pi^{+} \pi^{-} \eta}$ and (c) $M_{\pi^{+} \pi^{-}}$versus $M_{\pi^{+} \pi^{-} \eta}$ of the $D^{0} \rightarrow K_{S}^{0} \eta^{\prime}$ candidate events in data, where the regions between the pairs of solid (dashed) arrows denote the $K_{S}^{0}\left(\eta^{\prime}\right)$ signal (sideband) regions, and the solid, dashed and dotted boxes denote the signal, sideband A and sideband B regions (see text), respectively.

(sideband) regions. To estimate the non- $K_{S}^{0}$ and non- $\eta^{\prime}$ peaking backgrounds in $D^{0} \rightarrow K_{S}^{0} \eta^{\prime}$ decays, twodimensional (2D) signal and sideband regions, as shown in Fig. 3(c), are used. The solid box is the 2D signal region, where both of the $\pi^{+} \pi^{-}$and $\pi^{+} \pi^{-} \eta$ combinations lie in the $K_{S}^{0}$ and $\eta^{\prime}$ signal regions, respectively. The dashed (dotted) boxes indicate the 2D sideband A (B) regions, in which one (both) of the $\pi^{+} \pi^{-}$and $\pi^{+} \pi^{-} \eta$ combinations lie in the $K_{S}^{0}\left(\eta^{\prime}\right)$ sideband regions.

The yields of peaking backgrounds in the $K_{S}^{0}\left(\eta^{\prime}\right)$ sideband regions in data are obtained with similar fits to the corresponding $M_{\mathrm{BC}}$ distributions. For the decays with a $K_{S}^{0}$ or $\eta^{\prime}$ alone in the final status, the net signal yields $N_{\text {net }}$ are obtained according to

$$
N_{\text {net }}=N_{\text {sig }}-\frac{1}{2} N_{\text {sb }}
$$

where $N_{\text {sig }}$ and $N_{\text {sb }}$ are the observed numbers of events in the signal and sideband regions, respectively, as obtained in the fits. For the decay $D^{0} \rightarrow K_{S}^{0} \eta^{\prime}$, the net signal yield is estimated by

$$
N_{\text {net }}=N_{\text {sig }}-\frac{1}{2} N_{\mathrm{sbA}}+\frac{1}{4} N_{\mathrm{sbB}},
$$

where $N_{\mathrm{sbA}}$ and $N_{\mathrm{sbB}}$ denote the peaking background yield in the sideband regions $\mathrm{A}$ and $\mathrm{B}$, respectively. Studies show that the main contribution of $N_{\mathrm{sbA}}$ is from $\left.D^{0} \rightarrow K_{S}^{0} \pi^{+} \pi^{-} \eta\right|_{\text {non- } \eta^{\prime}}$, and the $N_{\mathrm{sbB}}$ is negligible.

\section{BRANCHING FRACTION}

The branching fraction of the $D \rightarrow P_{1} P_{2}$ decay is determined according to

$$
\mathcal{B}\left(D \rightarrow P_{1} P_{2}\right)=\frac{N_{\text {net }}}{2 \times N_{D \bar{D}}^{\text {tot }} \times \varepsilon \times \mathcal{B}_{\text {sub }}},
$$

where $N_{\text {net }}$ is the background-subtracted signal yields of the data; $N_{D \bar{D}}^{\text {tot }}$ is the total number of $D \bar{D}$ pairs, which is

TABLE I. Background-subtracted signal yields $\left(N_{\text {net }}\right)$ of $D \rightarrow P_{1} P_{2}$ decays, the efficiencies $(\varepsilon)$, the branching fractions measured in this work $(\mathcal{B})$ and the world average values $\left(\mathcal{B}_{\text {PDG }}\right)$. For $D^{0} \rightarrow P_{1} P_{2}$ decays, we include the correction factors of quantum coherence in $N_{\text {net }}$. The efficiencies $\varepsilon$ do not include the branching fractions of $\pi^{0}, \eta, K_{S}^{0}$ and $\eta^{\prime}$ decays.

\begin{tabular}{lcccc}
\hline \hline Mode & $N_{\text {net }}$ & $\epsilon(\%)$ & $\mathcal{B}\left(\times 10^{-3}\right)$ & $\mathcal{B}_{\text {PDG }}\left(\times 10^{-3}\right)$ \\
\hline$D^{+} \rightarrow \pi^{+} \pi^{0}$ & $10108 \pm 267$ & $49.0 \pm 0.3$ & $1.259 \pm 0.033 \pm 0.023$ & $1.24 \pm 0.06$ \\
$D^{+} \rightarrow K^{+} \pi^{0}$ & $1834 \pm 168$ & $48.2 \pm 0.4$ & $0.232 \pm 0.021 \pm 0.006$ & $0.189 \pm 0.025$ \\
$D^{+} \rightarrow \pi^{+} \eta$ & $11636 \pm 215$ & $47.0 \pm 0.3$ & $3.790 \pm 0.070 \pm 0.068$ & $3.66 \pm 0.22$ \\
$D^{+} \rightarrow K^{+} \eta$ & $439 \pm 72$ & $44.6 \pm 0.3$ & $0.151 \pm 0.025 \pm 0.014$ & $0.112 \pm 0.018$ \\
$D^{+} \rightarrow \pi^{+} \eta^{\prime}$ & $3088 \pm 83$ & $21.5 \pm 0.2$ & $5.12 \pm 0.14 \pm 0.024$ & $4.84 \pm 0.31$ \\
$D^{+} \rightarrow K^{+} \eta^{\prime}$ & $87 \pm 25$ & $18.8 \pm 0.2$ & $0.164 \pm 0.051 \pm 0.024$ & $0.183 \pm 0.023$ \\
$D^{+} \rightarrow K_{S}^{0} \pi^{+}$ & $93883 \pm 352$ & $51.4 \pm 0.2$ & $15.91 \pm 0.06 \pm 0.30$ & $15.3 \pm 0.6$ \\
$D^{+} \rightarrow K_{S}^{0} K^{+}$ & $17704 \pm 151$ & $48.5 \pm 0.1$ & $3.183 \pm 0.029 \pm 0.060$ & $2.95 \pm 0.15$ \\
$D^{0} \rightarrow \pi^{+} \pi^{-}$ & $21107 \pm 249$ & $66.0 \pm 0.3$ & $1.508 \pm 0.018 \pm 0.022$ & $1.421 \pm 0.025$ \\
$D^{0} \rightarrow K^{+} K^{-}$ & $56359 \pm 272$ & $62.8 \pm 0.3$ & $4.233 \pm 0.021 \pm 0.064$ & $4.01 \pm 0.07$ \\
$D^{0} \rightarrow K^{\mp} \pi^{ \pm}$ & $534135 \pm 759$ & $64.7 \pm 0.1$ & $38.98 \pm 0.06 \pm 0.51$ & $39.4 \pm 0.4$ \\
$D^{0} \rightarrow K_{S}^{0} \pi^{0}$ & $66552 \pm 302$ & $37.1 \pm 0.2$ & $12.39 \pm 0.06 \pm 0.27$ & $12.0 \pm 0.4$ \\
$D^{0} \rightarrow K_{S}^{0} \eta$ & $9485 \pm 126$ & $32.0 \pm 0.1$ & $5.13 \pm 0.07 \pm 0.12$ & $4.85 \pm 0.30$ \\
$D^{0} \rightarrow K_{S}^{0} \eta^{\prime}$ & $2978 \pm 61$ & $12.7 \pm 0.1$ & $9.49 \pm 0.20 \pm 0.36$ & $9.5 \pm 0.5$ \\
\hline \hline
\end{tabular}


$(8296 \pm 31 \pm 64) \times 10^{3}$ for $D^{+} D^{-}$and $(10597 \pm 28 \pm$ 89) $\times 10^{3}$ for $D^{0} \bar{D}^{0}$ [26]; $\varepsilon$ is the detection efficiency obtained by the MC simulation and does not include the branching fractions for the possible cascade decays, and $\mathcal{B}_{\text {sub }}$ denotes the product branching fractions [10] of the intermediate resonances $\pi^{0}, \eta, K_{S}^{0}$ and $\eta^{\prime}$ in the cascade decays.

The detection efficiency $\varepsilon$ is determined by analyzing the inclusive MC sample with the same analysis procedure as applied to the data, including the $M_{\mathrm{BC}}$ fit and the background estimation. Because of the relatively high backgrounds in the DCS decays of $D^{+} \rightarrow K^{+} \pi^{0}, K^{+} \eta$ and $K^{+} \eta^{\prime}$, their detection efficiencies are determined from MC samples of $\psi(3770) \rightarrow D^{+} D^{-}$in which one $D$ is forced to decay into a signal mode and the other decays generically. By fitting the $M_{\mathrm{BC}}$ distributions we obtain the net signal yield from the MC samples for each decay. The detection efficiency is obtained by dividing the net signal yield by the total number of the produced signal events. To better describe the data, the MC simulated efficiencies are corrected by the differences between data and MC simulation as discussed in Sec. V.

Inserting the values of $N_{\text {net }}, N_{D \bar{D}}^{\text {tot }}, \varepsilon$ and $\mathcal{B}_{\text {sub }}$ into Eq. (5), we obtain the branching fractions of the decays of interest, as listed in Table I. For the branching fractions measured in this work, the first uncertainty is statistical and the second one is systematic. By subtracting the branching fraction of DCS decay $D^{0} \rightarrow K^{+} \pi^{-}$[10] from that of $D^{0} \rightarrow K^{\mp} \pi^{ \pm}$, we obtain the branching fraction of $D^{0} \rightarrow K^{-} \pi^{+}$to be $(3.882 \pm 0.006 \pm 0.051) \%$.

\section{SYSTEMATIC UNCERTAINTY}

Table II summarizes the sources of the systematic uncertainties in the branching fraction measurements. The uncertainties are estimated relative to the measured branching fractions and are described below.

(1) $N_{D \bar{D}}^{\text {tot }}$ : The total number of $D \bar{D}$ pairs produced in data is cited from our previous work [26]. They are determined with a combined analysis in which both single-tag and double-tag events are used. Their uncertainties are included in our measurement.

(2) Tracking (PID) of $K^{+}\left(\pi^{+}\right)$: The tracking (PID) efficiencies for $K^{+}\left(\pi^{+}\right)$are studied by using doubletag $D \bar{D}$ hadronic events. Small differences in the tracking (PID) efficiencies of $K^{+}\left(\pi^{+}\right)$between data and MC simulation (denoted as data-MC differences) have been observed. To better describe the data, the MC-simulated efficiencies are corrected by the momentum-dependent data-MC differences for the $K^{+}$or $\pi^{+}$. Afterwards, the systematic uncertainty for tracking (PID) is assigned as $1.0 \%$ $(0.6 \%)$ for each pion from $\eta^{\prime}$ decays, and $0.3 \%$ $(0.3 \%)$ per track for the others.

(3) $K_{S}^{0}$ reconstruction: The $K_{S}^{0}$ reconstruction efficiency, including the tracking efficiency for charged pions, is studied with control samples of $J / \psi \rightarrow$ $K^{*}(892)^{\mp} K^{ \pm} \quad$ with $\quad K^{*}(892)^{ \pm} \rightarrow K_{S}^{0} \pi^{ \pm} \quad$ and $J / \psi \rightarrow \phi K_{S}^{0} K^{ \pm} \pi^{\mp}$. Small data-MC differences are found, as presented in Ref. [27]. We correct the MC efficiencies for these differences and assign a systematic uncertainty of $1.5 \%$ for each $K_{S}^{0}$.

(4) $\pi^{0}$ and $\eta$ reconstruction: The $\pi^{0}$ reconstruction efficiency is verified with double-tag hadronic events $D^{0} \rightarrow K^{-} \pi^{+}$and $K^{-} \pi^{+} \pi^{+} \pi^{-}$versus $\overline{D^{0}} \rightarrow$ $K^{-} \pi^{+} \pi^{0} \quad$ and $K_{S}^{0}\left(\pi^{+} \pi^{-}\right) \pi^{0}$. Small data-MC differences for the $\pi^{0}$ reconstruction efficiencies are found and are corrected to the MC simulation efficiencies. After corrections, the uncertainty for the $\pi^{0}$ reconstruction efficiency is taken as $1.0 \%$. The uncertainty for the $\eta$ reconstruction efficiency is assigned as $1.0 \%$, too.

(5) $\Delta E$ requirement and $M_{\mathrm{BC}}$ fit: The uncertainty from the $\Delta E$ requirement is investigated with alternative requirements of $3.5 \sigma_{\Delta E}$ or $4.0 \sigma_{\Delta E}$. The resultant largest changes in the branching fractions are assigned as the uncertainties. The uncertainty from the

TABLE II. Relative systematic uncertainties (in \%) in the branching fraction measurements.

\begin{tabular}{|c|c|c|c|c|c|c|c|c|c|c|c|c|c|c|}
\hline Source & $\pi^{+} \pi^{0}$ & $K^{+} \pi^{0}$ & $\pi^{+} \eta$ & $K^{+} \eta$ & $\pi^{+} \eta^{\prime}$ & $K^{+} \eta^{\prime}$ & $K_{S}^{0} \pi^{+}$ & $K_{S}^{0} K^{+}$ & $\pi^{+} \pi^{-}$ & $K^{+} K^{-}$ & $K^{\mp} \pi^{ \pm}$ & $K_{S}^{0} \pi^{0}$ & $K_{S}^{0} \eta$ & $K_{S}^{0} \eta^{\prime}$ \\
\hline$N_{D \bar{D}}^{\mathrm{tot}}$ & 0.9 & 0.9 & 0.9 & 0.9 & 0.9 & 0.9 & 0.9 & 0.9 & 0.9 & 0.9 & 0.9 & 0.9 & 0.9 & 0.9 \\
\hline Tracking of $K^{+}\left(\pi^{+}\right)$ & 0.3 & 0.3 & 0.3 & 0.3 & 2.3 & 2.3 & 0.3 & 0.3 & 0.6 & 0.6 & 0.6 & $\ldots$ & $\ldots$ & 2.0 \\
\hline PID of $K^{+}\left(\pi^{+}\right)$ & 0.3 & 0.3 & 0.3 & 0.3 & 1.5 & 1.5 & 0.3 & 0.3 & 0.6 & 0.6 & 0.6 & $\cdots$ & $\ldots$ & 1.2 \\
\hline$K_{S}^{0}$ reconstruction & $\cdots$ & $\cdots$ & $\ldots$ & $\ldots$ & $\ldots$ & $\ldots$ & 1.5 & 1.5 & $\cdots$ & $\cdots$ & $\cdots$ & 1.5 & 1.5 & 1.5 \\
\hline$\pi^{0}(\eta)$ reconstruction & 1.0 & 1.0 & 1.0 & 1.0 & 1.0 & 1.0 & $\cdots$ & $\cdots$ & $\cdots$ & $\cdots$ & $\cdots$ & 1.0 & 1.0 & 1.0 \\
\hline$\Delta E$ requirement & 0.1 & 1.2 & 0.6 & 2.6 & 0.5 & 3.8 & 0.3 & 0.2 & 0.5 & 0.6 & 0.2 & 0.4 & 0.4 & 0.4 \\
\hline$M_{\mathrm{BC}}$ fit & 0.9 & 1.7 & 0.5 & 8.5 & 1.8 & 13.3 & 0.2 & 0.2 & 0.6 & 0.4 & 0.1 & 0.2 & 0.4 & 0.4 \\
\hline Background estimation & $\cdots$ & 0.6 & $\cdots$ & $\cdots$ & 0.2 & 4.3 & 0.1 & 0.3 & $\cdots$ & $\cdots$ & $\cdots$ & 0.5 & 0.2 & 0.8 \\
\hline Quoted branching fractions & 0.0 & 0.0 & 0.5 & 0.5 & 1.7 & 1.7 & 0.1 & 0.1 & $\cdots$ & $\cdots$ & $\cdots$ & 0.1 & 0.5 & 1.7 \\
\hline MC statistics & 0.7 & 0.8 & 0.5 & 0.6 & 0.8 & 1.0 & 0.3 & 0.3 & 0.4 & 0.5 & 0.1 & 0.5 & 0.4 & 0.6 \\
\hline QC effects & $\cdots$ & $\cdots$ & $\ldots$ & $\ldots$ & $\cdots$ & $\ldots$ & $\cdots$ & $\cdots$ & 0.2 & 0.1 & 0.1 & 0.2 & 0.5 & 0.7 \\
\hline Total & 1.8 & 2.7 & 1.8 & 9.0 & 4.1 & 14.9 & 1.9 & 1.9 & 1.5 & 1.5 & 1.3 & 2.2 & 2.3 & 3.8 \\
\hline
\end{tabular}


$M_{\mathrm{BC}}$ fit is examined with different fit ranges $(1.8335,1.8865)$ or $(1.8395,1.8865) \mathrm{GeV} / c^{2}$, different end points of 1.8863 or $1.8867 \mathrm{GeV} / c^{2}$ for the ARGUS function, and different signal shapes with various requirements on the MC-truth matched signal shapes. The largest changes on the branching fractions with respect to the nominal results are treated as the corresponding systematic uncertainties.

(6) Background estimation: The uncertainty from the $K_{S}^{0}\left(\eta^{\prime}\right)$ sideband region is examined by changing the scale factors based on MC simulations and by shifting the $K_{S}^{0}\left(\eta^{\prime}\right)$ signal or sideband regions by $\pm 2 \mathrm{MeV} / c^{2}$. The maximum changes of the branching fractions with respect to the nominal results are assigned as the systematic uncertainties due to background estimation.

For the DCS decay of $D^{+} \rightarrow K^{+} \pi^{0}$, some peaking-like background from $D^{+} \rightarrow K_{S}^{0}\left(\rightarrow \pi^{0} \pi^{0}\right) \pi^{+}$is found. This background has not been modeled in the efficiency determination. The difference of the measured branching fractions of $D^{+} \rightarrow K^{+} \pi^{0}$ with and without considering this background, $0.6 \%$, is assigned as an uncertainty.

(7) Quoted branching fractions: The uncertainties in the quoted branching fractions for $\pi^{0} \rightarrow \gamma \gamma, \eta \rightarrow \gamma \gamma$, $K_{S}^{0} \rightarrow \pi^{+} \pi^{-}$and $\eta^{\prime} \rightarrow \pi^{+} \pi^{-} \eta$ are $0.03 \%, 0.51 \%$, $0.07 \%$ and $1.63 \%$ [10], respectively.

(8) MC statistics: The uncertainty in the efficiencies due to limited MC statistics is taken into account.

(9) Quantum coherence (QC) effects: Since $D^{0}$ and $\bar{D}^{0}$ are coherently produced in the process $e^{+} e^{-} \rightarrow$ $\psi(3770) \rightarrow D^{0} \bar{D}^{0}$, quantum correlation is considered with a method introduced in Ref. [28] when measuring the signal yields. The correction factors are included in the signal yields listed in Table I. The parameters are quoted from the PDG [10] and Heavy Flavor Averaging Group [29] and their uncertainties propagate to the branching fractions as systematic uncertainties.

Assuming all the uncertainty sources are independent, the quadratic sum of these uncertainties gives the total systematic uncertainty in the measurement of the branching fraction for each decay.

\section{SUMMARY}

By analyzing the data sample corresponding to an integrated luminosity of $2.93 \mathrm{fb}^{-1}$ taken at $\sqrt{s}=$ $3.773 \mathrm{GeV}$, we measured the absolute branching fractions for the two-body hadronic decays $D^{+} \rightarrow \pi^{+} \pi^{0}, K^{+} \pi^{0}, \pi^{+} \eta$,
$K^{+} \eta, \pi^{+} \eta^{\prime}, K^{+} \eta^{\prime}, K_{S}^{0} \pi^{+}, K_{S}^{0} K^{+}$, and $D^{0} \rightarrow \pi^{+} \pi^{-}, K^{+} K^{-}$, $K^{-} \pi^{+}, K_{S}^{0} \pi^{0}, K_{S}^{0} \eta, K_{S}^{0} \eta^{\prime}$. As shown in Table I, our results are consistent with the world average values within uncertainties and the branching fractions of $D^{+} \rightarrow \pi^{+} \pi^{0}$, $K^{+} \pi^{0}, \pi^{+} \eta, \pi^{+} \eta^{\prime}, K_{S}^{0} \pi^{+}, K_{S}^{0} K^{+}$and $D^{0} \rightarrow K_{S}^{0} \pi^{0}, K_{S}^{0} \eta, K_{S}^{0} \eta^{\prime}$ are determined with improved precision. The measured branching fractions for $D^{0} \rightarrow K_{S}^{0} \pi^{0}$ and $D^{+} \rightarrow K_{S}^{0} K^{+}$are consistent with those measured using a double-tag technique in our previous works [14], but with better precision. These results are useful for tests of theoretical calculations and provide a better understanding of SU(3)flavor symmetry breaking effects in hadronic decays of the $D$ mesons [2-5].

\section{ACKNOWLEDGMENTS}

The BESIII Collaboration thanks the staff of BEPCII and the IHEP computing center for their strong support. This work is supported in part by National Key Basic Research Program of China under Contract No. 2015CB856700; National Natural Science Foundation of China (NSFC) under Contracts No. 11475090, No. 11305180, No. 10975093, No. 11005061, No. 11235011, No. 11335008, No. 11425524, No. 11475107, No. 11625523, No. 11635010; the Chinese Academy of Sciences (CAS) Large-Scale Scientific Facility Program; the CAS Center for Excellence in Particle Physics (CCEPP); Joint Large-Scale Scientific Facility Funds of the NSFC and CAS under Contracts No. U1632109, No. U1332201, No. U1532257, No. U1532258; CAS under Contracts No. KJCX2-YW-N29, No. KJCX2-YW-N45; CAS Key Research Program of Frontier Sciences under Contract No. QYZDJ-SSW-SLH003; 100 Talents Program of CAS; National 1000 Talents Program of China; INPAC and Shanghai Key Laboratory for Particle Physics and Cosmology; German Research Foundation DFG under Contracts No. Collaborative Research Center CRC 1044, No. FOR 2359; Istituto Nazionale di Fisica Nucleare, Italy; Joint Large-Scale Scientific Facility Funds of the NSFC and CAS; Koninklijke Nederlandse Akademie van Wetenschappen (KNAW) under Contract No. 530-4CDP03; Ministry of Development of Turkey under Contract No. DPT2006K-120470; National Natural Science Foundation of China (NSFC); National Science and Technology fund; The Swedish Research Council; U.S. Department of Energy under Contracts No. DE-FG0205ER41374, No. DE-SC-0010118, No. DE-SC-0010504, No. DE-SC-0012069; University of Groningen (RuG) and the Helmholtzzentrum fuer Schwerionenforschung GmbH (GSI), Darmstadt; WCU Program of National Research Foundation of Korea under Contract No. R322008-000-10155-0. 
[1] L.-L. Chau and H.-Y. Cheng, Phys. Rev. D 36, 137 (1987); H.-Y. Cheng and C.-W. Chiang, Phys. Rev. D 81, 074021 (2010).

[2] K. Waikwok and S. Rosen, Phys. Lett. B 298, 413 (1993).

[3] Y. Grossman and D. J. Robinson, J. High Energy Phys. 04 (2013) 067.

[4] F.-S. Yu, X.-X. Wang, and C.-D. Lü, Phys. Rev. D 84, 074019 (2011).

[5] H.-N. Li, C.-D. Lü, and F.-S. Yu, Phys. Rev. D 86, 036012 (2012).

[6] D. Acosta et al. (CDF Collaboration), Phys. Rev. Lett. 94, 122001 (2005).

[7] B. Aubert et al. (BABAR Collaboration), Phys. Rev. D 74, 011107 (2006).

[8] H. Mendez et al. (CLEO Collaboration), Phys. Rev. D 81, 052013 (2010).

[9] E. Won et al. (Belle Collaboration), Phys. Rev. Lett. 107, 221801 (2011).

[10] C. Patrignani et al. (Particle Data Group), Chin. Phys. C 40, 100001 (2016).

[11] M. Ablikim et al. (BESIII Collaboration), Phys. Rev. D 91, 112015 (2015).

[12] M. Ablikim et al. (BESIII Collaboration), Phys. Rev. D 97, 052005 (2018).

[13] M. Ablikim et al. (BESIII Collaboration), Phys. Lett. B 765, 231 (2017).

[14] W. J. Zheng (BESIII Collaboration), Proc. Sci., CHARM2016 (2016) 075.
[15] M. Ablikim et al. (BESIII Collaboration), Chin. Phys. C 37, 123001 (2013); Phys. Lett. B 753, 629 (2016).

[16] M. Ablikim et al. (BESIII Collaboration), Nucl. Instrum. Methods Phys. Res., Sect. A 614, 345 (2010).

[17] S. Agostinelli et al. (GEANT4 Collaboration), Nucl. Instrum. Methods Phys. Res., Sect. A 506, 250 (2003).

[18] S. Jadach, B. Ward, and Z. Was, Phys. Rev. D 63, 113009 (2001).

[19] E. Kuraev and V. S. Fadin, Yad. Fiz. 41, 733 (1985) [Sov. J. Nucl. Phys. 41, 466 (1985)].

[20] E. Barberio and Z. Was, Comput. Phys. Commun. 79, 291 (1994).

[21] D. J. Lange, Nucl. Instrum. Methods Phys. Res., Sect. A 462, 152 (2001); R.-G. Ping, Chin. Phys. C 32, 599 (2008).

[22] K. Nakamura et al. (Particle Data Group), J. Phys. G 37, 075021 (2010) and 2011 partial update for the 2012 edition.

[23] J. C. Chen, G. S. Huang, X. R. Qi, D. H. Zhang, and Y. S. Zhu, Phys. Rev. D 62, 034003 (2000).

[24] M. Ablikim et al. (BESIII Collaboration), Phys. Lett. B 734, 227 (2014).

[25] H. Albrecht et al. (ARGUS Collaboration), Phys. Lett. B 229, 304 (1989).

[26] M. Ablikim et al., arXiv: 1803.06293.

[27] M. Ablikim et al. (BESIII Collaboration), Phys. Rev. D 92, 112008 (2015).

[28] D. Asner and W. Sun, Phys. Rev. D 73, 034024 (2006); 77, 019901 (2008).

[29] Y. Amhis et al. (Heavy Flavor Averaging Group), Eur. Phys. J. C 77, 895 (2017). 